\title{
Differentially expressed microRNA-218 modulates the viability of renal cell carcinoma by regulating BCL9
}

\author{
JIANMING WANG ${ }^{1 *}$, YULIN YING ${ }^{2 *}$, SHUMEI BO ${ }^{1}$, GUANGJUN LI $^{3}$ and FEI YUAN ${ }^{4}$ \\ ${ }^{1}$ Department of Urology, Linyi People's Hospital, Linyi, Shandong 276003; ${ }^{2}$ Department of Urology, Linshu People's Hospital, \\ Linyi, Shandong 276700; ${ }^{3}$ Department of Surgery, Luozhuang People's Hospital, Linyi, Shandong 276013; \\ ${ }^{4}$ Department of Surgery, Yushan Central Hospital of Linshu County, Yushan, Shandong 276709, P.R. China
}

Received May 16, 2015; Accepted May 3, 2016

DOI: $10.3892 / \mathrm{mmr} .2016 .5403$

\begin{abstract}
The present study assessed the expression profiles of numerous microRNAs (miRs; miR-141, miR-187, miR-206, miR-218, miR-335 and miR-204) in 25 pairs of renal cell carcinoma (RCC) tissue samples and adjacent non-cancerous tissue. Reverse transcription-quantitative polymerase chain reaction (RT-qPCR) analysis revealed that miR-218 was significantly downregulated in RCC tissue samples. Next, web-based algorithms were used to identify B-cell lymphoma (BCL)9 as a possible target of miR-218, which was confirmed by a subsequent luciferase assay. Furthermore, the mRNA expression of BCL9 in the tumor and adjacent normal tissue samples was assessed, and BCL9 was markedly upregulated in the tumor tissue samples compared with the adjacent non-cancerous controls. In addition, miR-218 mimics or its inhibitors were transfected into renal cell carcinoma cells and harvested $48 \mathrm{~h}$ later. RT-qPCR and western blotting revealed that both the mRNA and protein expression levels of BCL9 were significantly downregulated by the miR-218 mimics. However, inhibition of miR-218 upregulated the expression levels of BCL9. A 3-(4,5-dimethylthiazol-2-yl)-2,5-diphenyltetrazolium bromide (MTT) proliferation assay revealed that cell proliferation was suppressed in miR-218 mimic-transfected RCC cells compared with control cells; however, cell proliferation was significantly promoted in the RCC cells transfected with miR-218 inhibitors compared with the controls. Taken
\end{abstract}

Correspondence to: Dr Guangjun Li, Department of Surgery, Luozhuang People's Hospital, 123 Shuangyueyuan Road, Linyi, Shandong 276013, P.R. China

E-mail: guangjun_1@163.com

Dr Fei Yuan, Department of Surgery, Yushan Central Hospital of Linshu County, 18 Cangsheng Road, Linshu County, Yushan, Shandong 276709, P.R. China

E-mail: feifei_1f@163.com

*Contributed equally

Key words: microRNA-218, proliferation, renal cell carcinoma, BCL9, apoptosis together, it was demonstrated that miR-218 modulated a novel molecular target and the present study provided novel insights into potential mechanisms of RCC oncogenesis.

\section{Introduction}

Renal cell carcinoma (RCC) is the most frequent neoplasm that occurs in the adult kidney (1), and the 5 year survival rates are $98 \%$ for stage I disease and $50 \%$ for stage III disease (2). These survival rates strengthen the significance of early identification and timely treatment of RCC. The mortality rate has not been substantially improved in the past two decades, although certain progress has been achieved in early recognition of RCC $(3,4)$. RCC is characterized by aberrantly enhanced proliferation of the cancer cells, and as a result, $\mathrm{RCC}$ is relatively resistant to the conventional treatment of the disease, including radiotherapy and chemotherapy (5). Although options are available in the management of RCC, the clinical outcome is usually limited due to its fast growth (4). Therefore, an improved understanding of the underlying molecular mechanism and identification of novel and pivotal diagnostic and therapeutic molecules are warranted.

A growing body of evidence demonstrated that micro (mi)RNAs, a class of non-protein-coding small RNAs, are associated with the progression and proliferation of cancer. miRNAs post-transcriptionally modulate gene expression via binding to the 3'-untranslated region (UTR) of target mRNAs, resulting in suppression of translation and/or degradation of mRNA (6). miRNAs may be differentially expressed between normal and tumor tissue, and miRNA expression profiles may be specific in certain cancer types, which may be responsible for the development of malignancy, including RCC (7). Discovery of differentially expressed miRNA and the identification its downstream signaling pathway may provide the possibility to develop novel therapeutic methods for the treatment of RCC.

It has been previously reported that miRNAs are responsible for the abnormally enhanced proliferation of RCC cells, and miR-141, miR-187, miR-206, miR-218, miR-335 and miR-204 have been identified to be differentially expressed in RCC based on miRNA microarray analysis (8). The present study performed confirmatory reverse transcription-quantitative polymerase chain reaction (RT-qPCR) to identify the expression level of each of those miRNAs in RCC tumor 
tissue sample compared with the adjacent non-cancerous control tissue samples. The results revealed that miR-218 was significantly downregulated in RCC tissue. The present study also identified B-cell lymphoma (BCL)9 as a target of miR-218 by computational analysis, which was confirmed by luciferase assay.

\section{Materials and methods}

Patients and sample collection. The present study was approved by the Ethics Committee of Yushan Central Hospital of Linshu County, (Yushan, China). Written informed consent was obtained from all patients. A total of 25 pairs of tumor tissue samples and adjacent non-cancerous tissue samples, at least $2 \mathrm{~cm}$ away from the tumor tissue, were collected from patients who were diagnosed with RCC and received nephron-sparing surgery or radical nephrectomy for RCC at the Department of Surgery, Yushan Central Hospital of Linshu County. Patients treated with chemotherapy or radiotherapy prior to surgery were excluded from the present study. Soon after removal, the tissue samples were frozen in liquid nitrogen for future use.

$R T-q P C R$. The mirVana ${ }^{\mathrm{TM}}$ miRNA isolation kit (Ambion, USA) was used to extract the total RNA from the tissues or the cells, according to the manufacturer's protocol. A NanoDrop spectrophotometer (ND-1000; Thermo Fisher Scientific, Inc., Waltham, MA, USA) was used to identify the quantity and purity of the RNA. The RNA samples were maintained at $-80^{\circ} \mathrm{C}$ for future use. The synthesis of cDNA from the total RNA was performed by using miRNA-specific RT primers from the TaqMan MicroRNA assay (Applied Biosystems, Foster City, CA, USA) at $65^{\circ} \mathrm{C}$ for $5 \mathrm{~min}, 25^{\circ} \mathrm{C}$ for $10 \mathrm{~min}$, $42^{\circ} \mathrm{C}$ for $1 \mathrm{~h}$ and $75^{\circ} \mathrm{C}$ for $10 \mathrm{~min}$. Subsequently, using the TaqMan MicroRNA assay, qPCR was performed on the ABI PRISM 7900HT system (Applied Biosystems). The conditions were as follows: $95^{\circ} \mathrm{C}$ for 5 min followed by 40 cycles of $95^{\circ} \mathrm{C}$ for $10 \mathrm{sec}$ and $60^{\circ} \mathrm{C}$ for $60 \mathrm{sec}$. Each experiment was repeated at least three times. The primer sequences were as follows: Forward, 5'-TCAGTTTGCTGTTCTGGGTG-3' and reverse, 5'-CGGTTGGCTGGAAAGGAG-3' for GAPDH, and forward, 5'-GAGCACTGCAGTGTCGTGAC-3' and reverse, 5'-TCCCCTTGCCCTTGGCACCA-3' for the miRNA-specific primers. SDS software (version 2.4; Applied Biosystems; Thermo Fisher Scientific, Inc.) was used to perform quantitative analysis according to the manufacturer's protocol. The software dissociation curve analysis feature was used to verify and determine the specificity of the PCR products. The $2^{-\Delta \Delta C q}$ method was used to analyze the relative quantification, as described previously (9).

Oligonucleotides and transfection. The miR-218 mimics, miR-218 inhibitor and oligonucleotide negative control (NC) miRNA were obtained from GenePharma (Shanghai, China). The transfection of oligonucleotides were performed using Lipofectamine 2000 (Invitrogen; Thermo Fisher Scientific, Inc.), according to the manufacturer's protocol. The A498 cell line was purchased from American Type Culture Collection (Manassas, VA, USA), and the cells were incubated in each well of a 96-well plate overnight.
Colorimetric 3-(4,5-dimethylthiazol-2-yl)-2,5-diphenyltetrazolium bromide (MTT) assay. To investigate cell proliferation, MTT (Promega Corporation, Madison, WI, USA) was pipetted into all wells at different time points. The cells were cultured at $37.8^{\circ} \mathrm{C}$ for $3 \mathrm{~h}$. Subsequently, the cells were centrifuged at $13,000 \mathrm{x} \mathrm{g}$ for $15 \mathrm{~min}$ at $4^{\circ} \mathrm{C}$ to remove cell debris and insoluble material. A total of $100 \mu \mathrm{l}$ dimethyl sulfoxide was used to dissolve the formazan precipitates. A spectrophotometer (Dinex Technologies, Chantilly, VA, USA) was used to measure the absorbance at a wavelength of $550 \mathrm{~nm}$.

Western blotting. Following washing with cold phosphate-buffered saline, the cells were lysed in radioimmunoprecipitation buffer (BioTeke Corporation, Beijing, China) comprising $5 \mathrm{mM}$ EDTA, $150 \mathrm{mM} \mathrm{NaCl}, 50 \mathrm{mM}$ Tris $\mathrm{Cl}$ (pH 7.4), 0.1\% sodium dodecyl sulfate (SDS)/1\% aprotinin, $1 \%$ sodium deoxycholate, $1 \%$ Nonidet P-40, $0.1 \mathrm{mM} \mathrm{Na}_{3} \mathrm{VO}_{4}$ and $50 \mathrm{mM} \mathrm{NaF}$, supplemented with a Protease Inhibitor Cocktail (Promega Corporation) on ice for $30 \mathrm{~min}$. Following protein extraction, the samples were centrifuged at $13,000 \mathrm{xg}$ for $15 \mathrm{~min}$ at $4^{\circ} \mathrm{C}$. A bicinchoninic acid (BCA) assay kit (BioTeke Corporation) was used to determine the concentration of the protein, according to the manufacturer's protocol. The proteins were heated with loading buffer (BioTeke Corporation) at $95.8^{\circ} \mathrm{C}$ for $5 \mathrm{~min}$, and were separated by using $10 \%$ SDS-polyacrylamide gel electrophoresis. The proteins were transferred onto a polyvinylidene difluoride membrane (Bio-Rad Laboratories, Hercules, CA, USA). The membrane was blocked in blocking buffer (Merck Millipore, Darmstadt, Germany) with 5\% skim milk for $60 \mathrm{~min}$ at room temperature and subsequently incubated overnight at $4^{\circ} \mathrm{C}$ with mouse monoclonal antibodies against $\beta$-actin (1:2,000; Santa Cruz Biotechnology, Inc., Santa Cruz, CA, USA; cat. no. sc-47778) and BCL9 (1:1,000; Santa Cruz Biotechnology, Inc.; cat. no. sc-81199). Following primary antibody incubation, the membranes were incubated with goat anti-mouse horseradish peroxidase-conjugated secondary antibody (1:3,000; Santa Cruz Biotechnology, Inc.; cat. no. sc-2005) for $1 \mathrm{~h}$ at room temperature. The percentage of the expression of target proteins was normalized against $\beta$-actin using ImageJ software (imagej.nih.gov/ij).

Luciferase assay. A498 cells were seeded into a 12-well plate at a density of $\sim 0.8 \times 10^{5}$ cells for $24 \mathrm{~h}$. The cells were subsequently transfected with $100 \mathrm{nmol} / \mathrm{l} \mathrm{miR}-218$ mimics using Lipofectamine 2000 (Invitrogen; Thermo Fisher Scientific, Inc.) and $50 \mathrm{ng}$ target sequence (wild type or mutant BCL9 3'-UTR) expression clone (pGL3-BCL9 3'UTR). After incubation for $36 \mathrm{~h}$, a luciferase assay system (Promega Corporation) was used to determine the activities of firefly luciferase, according to the manufacturer's protocol. An LD400 luminometer (Beckman Coulter, Brea, CA, USA) was used to collect data.

Apoptosis assay. Transfection was performed when cell confluency reached $80 \%$ in the 48 -well plates. The cells were serum starved and $10 \mu \mathrm{M}$ camptothecin (Sigma-Aldrich) in serum-free media was used to induce apoptosis as previously described (10). Propidium iodide (Invitrogen; Thermo Fisher Scientific, Inc.) and Annexin V-fluorescein isothiocyanate (Invitrogen; Thermo Fisher Scientific, Inc.) were used to stain 


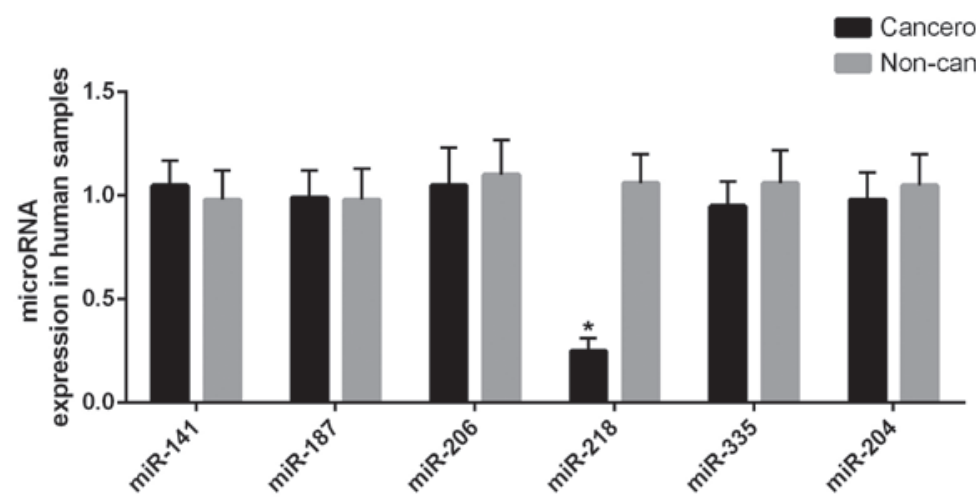

Figure 1. Differences in miRNA expression levels in cancerous and non-cancerous tissues. The expression levels of miR-141, miR-187, miR-206, miR-218, miR-335 and miR-204 were assessed in renal cell carcinoma and non-cancerous adjacent tissues. miR-218 was revealed to be significantly downregulated in cancerous tissue tissues compared with the non-cancerous tissues. The data are expressed as the mean \pm standard deviation ("P $<0.01$ vs. the non-cancerous tissue). miR, microRNA.

Hsa-miR-218 targets BCL9

Hsa-miR-218

3' -UGUACCAAUCUAGUUCGUGUU-5'

Hsa-miR-218

Wild-type BCL9 3'UTR 5' -GCAGAGTATTAATGAAAAGCACAA-3'

Mutant BCL9 3'UTR

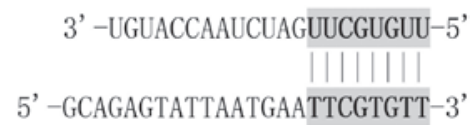

Figure 2. A putative binding site of miR-218 was identified in the 3'UTR of BCL9, and the binding site was replaced with complimentary sequences. miR, microRNA; Hsa, Homo sapiens; UTR, untranslated region.

the cells, and FACScan flow cytometer (BD Biosciences, Franklin Lakes, NJ, USA) was used to analyze the apoptosis of A498 cells, and CellQuest Software (BD Biosciences) was used to analyze the data according to the manufacturer's protocol. Each test was repeated three times.

Statistical analysis. All statistical analyses were performed using SPSS 21.0 (IBM SPSS, Chicago, IL, USA). The data are expressed as the mean \pm standard deviation. The comparison between two groups was assessed using an independent $\mathrm{t}$-test, and the comparison among multiple groups ( $>2$ groups) were performed by using one-way analysis of variance. $\mathrm{P}<0.05$ was considered to indicate a statistically significant difference.

\section{Results}

It has been previously reported that miRNAs are responsible for the abnormally enhanced proliferation of RCC cells (8). The present study was focused on the differential expression of miRNAs, as well as its role in tumorigenesis of RCC. A total of 25 pairs of tumor tissue samples and adjacent non-cancerous controls were obtained from patients with confirmed diagnosis of RCC. RT-qPCR was performed for the tissue samples to determine and compare the expression of miRNAs (miR-141, miR-187, miR-206, miR-218, miR-335 and miR-204) (8). miR-218 was revealed to be significantly $(\mathrm{P}<0.01)$ downregulated in the tumor tissue compared with the controls, as shown in Fig. 1.

The present study used online publicly available algorithms (mirdb.org) to predict targets of miR-218. The results revealed the putative binding site of miR-218 in the 3'UTR of BCL9 (Fig. 2). To confirm this, the present study constructed pGL3-BCL9-3'UTR, containing the miR-218 binding site or

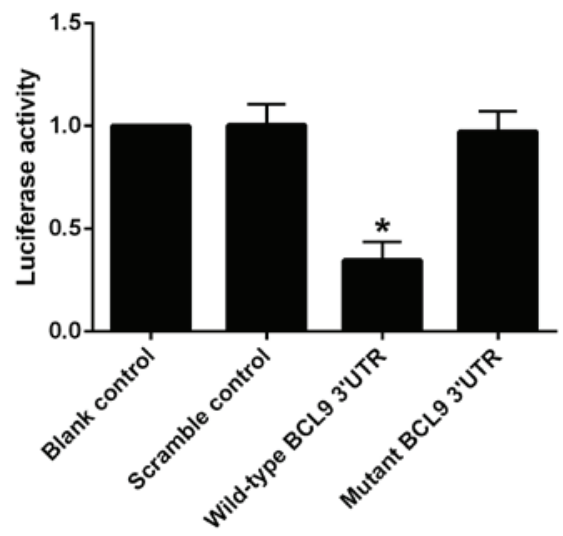

Figure 3. Differences in luciferase activity in response to various transfections. The luciferase activity of the wild-type pGL3-BCL9-3'UTR transfected into A498 cells was significantly inhibited by miR-218, however, was unaffected by the scramble control. Additionally, the luciferase activity of the mutant pGL3-BCL9-3'UTR was unaffected by transfection of miR-218. The data are expressed as the mean \pm standard deviation. ${ }^{*} \mathrm{P}<0.05$ vs. the scramble control. miR, microRNA; UTR, untranslated region.

the mutant binding site (Fig. 2), and co-transfected the RCC cells with the construct and miR-218 mimics or scramble controls. As showed in Fig. 3, the luciferase activity from pGL3-BCL9-3'UTR was significantly inhibited by miR-218, but was not affected by the scramble controls. These findings indicated that miR-218 inhibited the expression of BCL9 by binding to complementary sequences in the 3'UTR of BCL9.

Given the negative regulatory association between miR-218 and BCL9 in RCC cells, the present study further examined the expression of BCL9 in the tumor samples and the controls, finding that the mRNA expression of BCL9 was substantially upregulated in the tumor tissue samples 

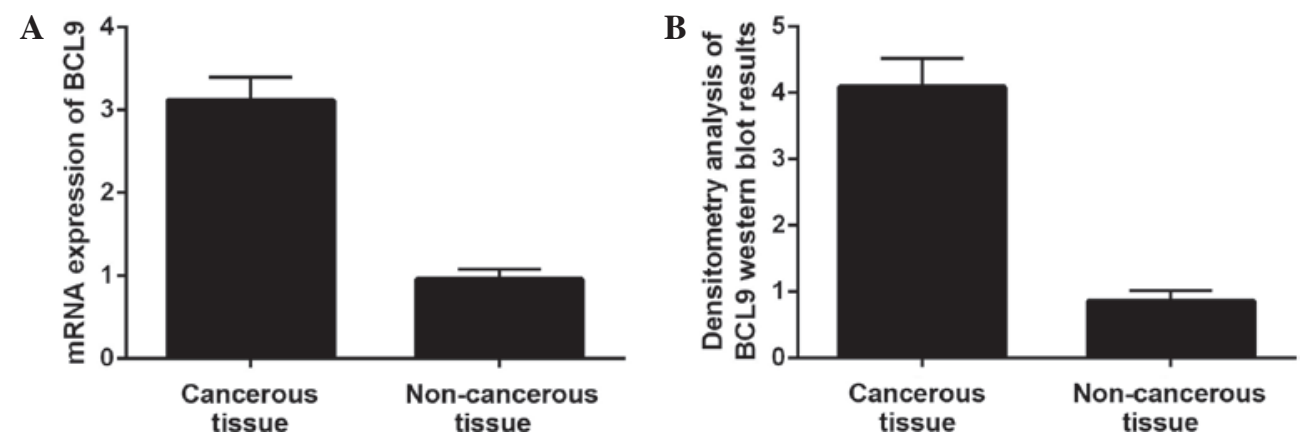

Figure 4. BCL9 expression in cancerous and non-cancerous tissue. The (A) mRNA and (B) protein expression levels of BCL9 were notably upregulated in the renal cell carcinoma tissue samples as compared with the adjacent non-cancerous tissues, as indicated by reverse transcription-quantitative polymerase chain reaction and western blotting, respectively. The data are expressed as the mean \pm standard deviation.

A

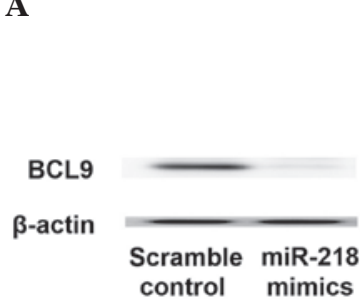

A

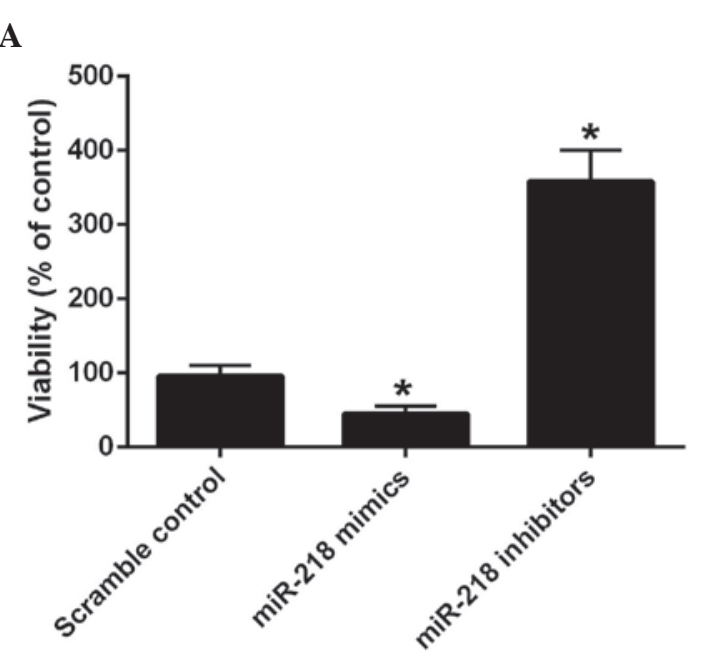
${ }^{*} \mathrm{P}<0.05$ vs. the scramble control. si, small interfering; miR, microRNA.
B

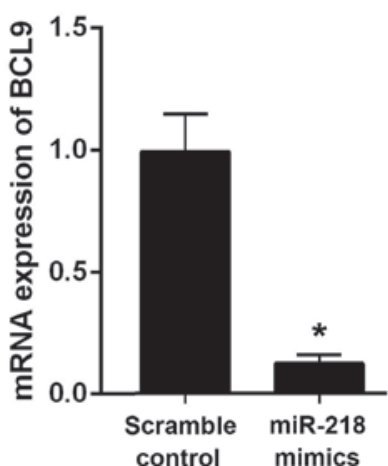

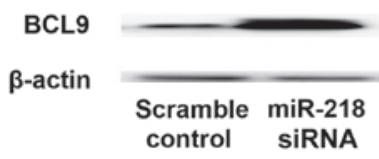

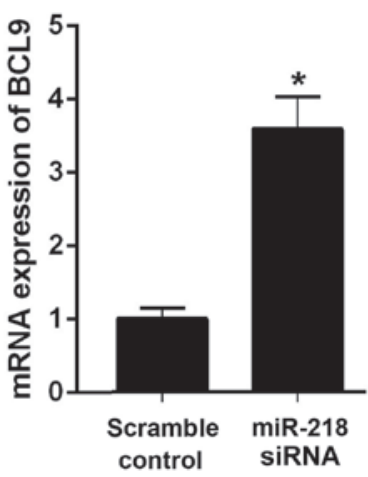

Figure 5. BCL9 expression in response to miRNA mimics. (A) The protein and mRNA expression levels of BCL9 was significantly downregulated by the miR-218 mimics in A498 cells. (B) Following the reduction of miR-218 following siRNA transfection, BCL9 was significantly upregulated. Representative western blots and reverse transcription-quantitative polymerase chain reaction results are shown. The data are expressed as the mean \pm standard deviation.
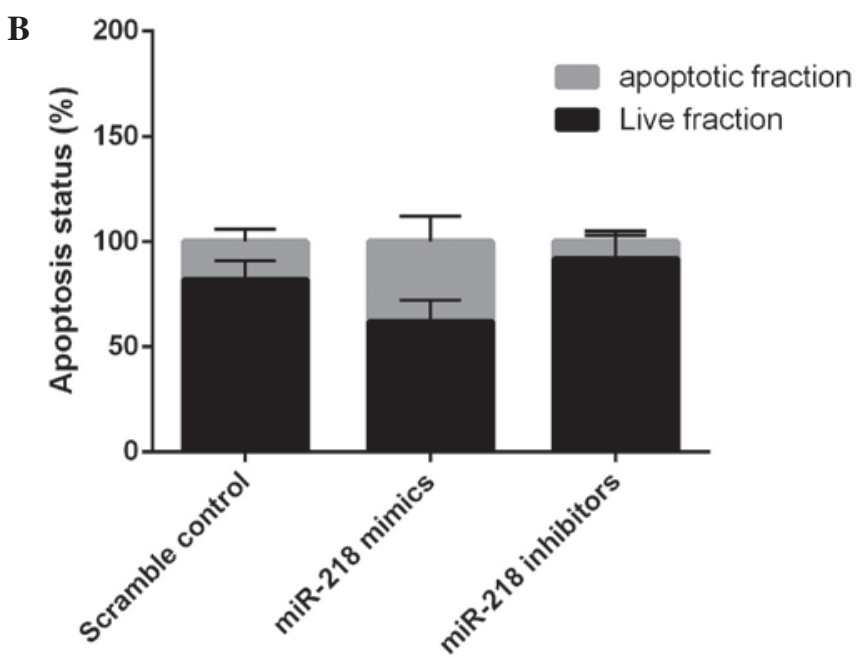

Figure 6. Cell viability and apoptosis are affected by miR-218. (A) Cell viability was reduced in miR-218 mimic-transfected RCC cells compared with control cells, while the viability was significantly enhanced in the RCC cells transfected with miR-218 inhibitors. (B) miR-218 overexpression increased the apoptotic status of the RCC cells, while miR-218 inhibitors significantly suppressed apoptosis in RCC cells compared with the control. The data are expressed as the mean \pm standard deviation. " $\mathrm{P}<0.05$ vs. the scramble control. miR, microRNA; RCC, renal cell carcinoma.

compared with the adjacent non-cancerous controls (Fig. 4A). In addition, western blot analysis was performed to determine the protein expression levels of BCL9, and ImageJ software was used to evaluate the relative density of the target bands normalized against $\beta$-actin. As shown in Fig. 4B, the protein expression of BCL9 was significantly higher in the cancerous tissue compared with the non-cancerous controls. To further study whether miR-218 affects the expression of BCL9, miR-218 mimics or its inhibitors were transfected into RCC cells and were harvested $48 \mathrm{~h}$ later. RT-qPCR and western blotting revealed that both the mRNA and protein expression levels of BCL9 were significantly downregulated 
by the miR-218 mimics (Fig. 5A). However, the inhibition of miR-218 upregulated BCL9 levels (Fig. 5B).

Next, the present study explored the potential impact of miR-218 on the proliferation of RCC cells. The cells were transfected with the miR-218 mimics, inhibitors or the scramble controls, and were analyzed $48 \mathrm{~h}$ later. An MTT proliferation assay revealed that cell proliferation was suppressed in miR-218 mimic-transfected RCC cells compared with the control cells; however, cell proliferation was significantly promoted in the RCC cells transfected with miR-218 inhibitors compared with the controls (Fig. 6A). Subsequently, apoptosis analysis revealed that miR-218 overexpression caused more cell death in RCC cells compared with the NC cells, while introduction of miR-218 inhibitors significantly suppressed the apoptosis in RCC cells compared with the control cells (Fig. 6B).

\section{Discussion}

Dysregulation of numerous miRNAs in RCC were observed in several cancer types, demonstrating that miRNAs have a regulatory role in ordinary cancer pathways. miRNAs can act on genes that are associated with cancer pathogenesis and their actions as tumor inhibitor genes or oncogenes has been demonstrated previously (5). An improved knowledge of the effects of miRNAs in these cellular processes may assist with the development of novel therapies for the treatment of cancer. In the present study, 25 pairs of tumor tissue samples and adjacent non-cancerous controls were obtained from patients with confirmed diagnosis of RCC. RT-qPCR was performed to determine and compare the expression levels of miR-141, miR-187, miR-206, miR-218, miR-335 and miR-204 (8). miR-218 was significantly downregulated in the tumor tissue samples compared with the controls, as shown in Fig. $1(\mathrm{P}<0.01)$.

The role of miR-218 has been well studied, and the data from previous functional studies of miR-218 in a variety of cancer types revealed the inhibition of cancer cell proliferation and invasion by miR-218. For instance, underexpression of miR-218 was revealed to be correlated with lymph node metastasis, advanced clinical stage and unfavorable prognosis (11). In non-small cell lung carcinoma, the expression of miR-218 may act as an independent signal for relapse-free and overall survival (12). Numerous previous studies have successfully identified direct targets of miR-218, including asIKK-B, ECOP, PXN, LASP1, Survivin, RICTOR and ROBO1 (13-17). These findings indicated that the expression of miR-218 may be an effective indicator for tumor diagnosis and prognosis, and miR-218 acts as a tumor inhibitor in certain other cancer types. In the present study, online publicly available algorithms (mirdb.org) were used to predict miR-218 targets. A putative binding site of miR-218 was identified in the 3'UTR of BCL9. To confirm this regulatory association, the present study constructed pGL3-BCL9-3'UTR, containing the miR-218 binding site or the mutant one, and co-transfected the RCC cells with the construct and miR-218 mimics or scramble controls. It was revealed that the luciferase activity from pGL3-BCL9-3'UTR was significantly inhibited by miR-218, but was not affected by the scramble controls. These findings indicated that
miR-218 inhibits the expression of BCL9 by binding to complementary sequences in the 3'UTR of BCL9.

BCL9 is a crucial co-mediator of the transcriptional activity of $\mathrm{Wnt} / \beta$-catenin, and previous studies have demonstrated its function as novel therapeutic target of malignancies $(18,19)$. Previous studies have demonstrated that BCL9 functions as oncogene, which can promote tumor progression (epithelial-mesenchymal transition, invasion, migration and metastasis) and cell proliferation by regulating the expression of cyclin D1. By contrast, knocking down BCL9 improves survival outcomes in xenograft mouse models of multiple myeloma and colon cancer (18). Notably, knocking out BCL9 in germ cells increased apoptotic cells by 10-fold (18). BCL9 has also been previously reported to be regulated by miRNAs. For instance, miR-30 was revealed to be involved in the control of BCL9 in multiple myeloma (MM), and a reverse correlation exists between miR-30s and the mRNA expression levels of BCL9 (20). Elevated expression of miR-30s in MM cell lines results in a decrease in proliferation, migration, survival, invasion and colony formation (21). In the present study, it was revealed that the protein expression of BCL9 was significantly higher in the cancerous tissue compared with the non-cancerous controls, and both the mRNA and protein expression levels of BCL9 were significantly downregulated by miR-218 mimics. However, inhibition of miR-218 upregulated BCL9 levels.

Furthermore, the present study assessed the potential impact of miR-218 in RCC cell proliferation. The cells were transfected with miR-218 mimics, inhibitors or the scramble controls, and were analyzed $48 \mathrm{~h}$ later. An MTT proliferation assay demonstrated that cell proliferation was suppressed in miR-218 mimic-transfected RCC cells compared with the control cells; however, cell proliferation was significantly promoted in the RCC cells transfected with miR-218 inhibitors compared with the controls. Subsequent apoptosis analysis revealed that miR-218 overexpression caused increased death to RCC cells compared with NC cells, while introduction of miR-218 inhibitors significantly suppressed the apoptosis in RCC cells compared with the control.

In conclusion, significant downregulation of miR-218 was observed in RCC cells. The expression of miR-218 was decreased in clinical specimens of RCC, and had an inhibitory role in the control of proliferation of RCC. The present results revealed that oncogenic BCL9 may be upregulated as a result of the downregulation of tumor suppressive miR-218 in the progression of human RCC. This molecular network is likely important in oncogenesis of RCC and may act as a novel therapeutic option for patients with RCC.

\section{References}

1. Weng L, Wu X, Gao H, Mu B, Li X, Wang JH, Guo C, Jin JM, Chen Z, Covarrubias M, et al: MicroRNA profiling of clear cell renal cell carcinoma by whole-genome small RNA deep sequencing of paired frozen and formalin-fixed, paraffin-embedded tissue specimens. J Pathol 222: 41-51, 2010.

2. Devita VT Jr, Hellman S and Rosenberg SA (eds): Cancer principles and practice of oncology. 8th edition. Lippincott Williams \& Wilkins, Philadelphia, PA, 2008.

3. Fridman E, Dotan Z, Barshack I, David MB, Dov A, Tabak S, Zion O, Benjamin S, Benjamin $\mathrm{H}$, Kuker $\mathrm{H}$, et al: Accurate molecular classification of renal tumors using microRNA expression. J Mol Diagn 12: 687-696, 2010. 
4. Aben KK, Luth TK, Janssen-Heijnen ML, Mulders PF, Kiemeney LA and van Spronsen DJ: No improvement in renal cell carcinoma survival: A population-based study in the Netherlands. Eur J Cancer 44: 1701-1709, 2008.

5. Capitanio U, Cloutier V, Zini L, Isbarn H, Jeldres C, Shariat SF Perrotte P, Antebi E, Patard JJ, Montorsi F and Karakiewicz PI: A critical assessment of the prognostic value of clear cell, papillary and chromophobe histological subtypes in renal cell carcinoma: A population-based study. BJU Int 103: 1496-1500, 2009.

6. Slaby O, Jancovicova J, Lakomy R, Svoboda M, Poprach A, Fabian P, Kren L, Michalek J and Vyzula R: Expression of miRNA-106b in conventional renal cell carcinoma is a potential marker for prediction of early metastasis after nephrectomy. J Exp Clin Cancer Res 29: 90, 2010.

7. Volinia S, Calin GA, Liu CG, Ambs S, Cimmino A, Petrocca F Visone R, Iorio M, Roldo C, Ferracin M, et al: A microRNA expression signature of human solid tumors defines cancer gene targets. Proc Natl Acad Sci USA 103: 2257-2261, 2006.

8. Hidaka H, Seki N, Yoshino H, Yamasaki T, Yamada Y, Nohata N, Fuse M, Nakagawa $M$ and Enokida $H$ : Tumor suppressive microRNA-1285 regulates novel molecular targets: Aberrant expression and functional significance in renal cell carcinoma. Oncotarget 3: 44-57, 2012.

9. Vermes I, Haanen C, Steffens-Nakken H and Reutelingsperger C: A novel assay for apoptosis. Flow cytometric detection of phosphatidylserine expression on early apoptotic cells using fluorescein labelled Annexin V. J Immunol Methods 184: 39-51.

10. Annaratone L, Volante M, Asioli S, Rangel N and Bussolati G: Characterization of neuroendocrine tumors of the pancreas by real-time quantitative polymerase chain reaction. A methodological approach. Endocr Pathol 24: 83-91, 2013.

11. Tie J, Pan Y, Zhao L, Wu K, Liu J, Sun S, Guo X, Wang B, Gang Y,Zhang Y, et al: MiR-218 inhibits invasion and metastasis of gastric cancer by targeting the Robol receptor. PLoS Genet 6: e1000879, 2010

12. Smilenov LB, Mikhailov A, Pelham RJ, Marcantonio EE and Gundersen GG: Focal adhesion motility revealed in stationary fibroblasts. Science 286: 1172-1174, 1999.
13. Alajez NM, Lenarduzzi M, Ito E, Hui AB, Shi W, Bruce J, Yue S, Huang SH, Xu W, Waldron J, et al: MiR-218 suppresses nasopharyngeal cancer progression through downregulation of survivin and the SLIT2-ROBO1 pathway. Cancer Res 71: 2381-2391, 2011.

14. Chiyomaru T, Enokida H, Tatarano S, Kawahara K, Uchida Y, Nishiyama K, Fujimura L, Kikkawa N, Seki N and Nakagawa M: miR-145 and miR-133a function as tumour suppressors and directly regulate FSCN1 expression in bladder cancer. Br J Cancer 102: 883-891, 2010.

15. Song L, Huang Q, Chen K, Liu L, Lin C, Dai T, Yu C, Wu Z and Li J: miR-218 inhibits the invasive ability of glioma cells by direct downregulation of IKK- $\beta$. Biochem Biophys Res Commun 402: 135-140, 2010.

16. Wu DW, Cheng YW, Wang J, Chen CY and Lee H: Paxillin predicts survival and relapse in non-small cell lung cancer by microRNA-218 targeting. Cancer Res 70: 10392-10401, 2010.

17. Gao C, Zhang Z, Liu W, Xiao S, Gu W and Lu H: Reduced microRNA-218 expression is associated with high nuclear factor kappa B activation in gastric cancer. Cancer 116: 41-49, 2010

18. Mani M, Carrasco DE, Zhang Y, Takada K, Gatt ME, Dutta-Simmons J, Ikeda H, Diaz-Griffero F, Pena-Cruz V, Bertagnolli M, et al: BCL9 promotes tumor progression by conferring enhanced proliferative, metastatic and angiogenic properties to cancer cells. Cancer Res 69: 7577-7586, 2009.

19. Takada K, Zhu D, Bird GH, Sukhdeo K, Zhao JJ, Mani M, Lemieux M, Carrasco DE, Ryan J, Horst D, et al: Targeted disruption of the BCL9/ $\beta$-catenin complex inhibits oncogenic Wnt signaling. Sci Transl Med 4: 148ra117, 2012.

20. Nantel F, Monaco L, Foulkes NS, Masquilier D, LeMeur M, Henriksén K, Dierich A, Parvinen M and Sassone-Corsi P: Spermiogenesis deficiency and germ-cell apoptosis in CREM-mutant mice. Nature 380: 159-162, 1996.

21. Zhao JJ, Lin J, Zhu D, Wang X, Brooks D, Chen M, Chu ZB, Takada K, Ciccarelli B, Admin S, et al: miR-30-5p functions as a tumor suppressor and novel therapeutic tool by targeting the oncogenic Wnt/ $\beta$-catenin/BCL9 pathway. Cancer Res 74: 1801-1813, 2014. 